\title{
The Decay of the Gravitational Field
}

\author{
B. G. Schmidt
}

Max-Planck-Institut für Physik und Astrophysik, Institut für Astrophysik, D-8046 Garching, Federal Republic of Germany

\begin{abstract}
The asymptotic behaviour of Einstein-Rosen waves, a class of nonstationary solutions of Einstein's vacuum equations, is investigated. It is established that solutions of this type exist which admit part of $\mathscr{I}^{+}$and a regular $I^{+}$in the sense of Penrose.
\end{abstract}

\section{Introduction}

In classical field theories it is important to know how the field decays. Does the free field at a fixed space point always ultimately tend to zero if time goes to infinity? Are there soliton-like solutions? Answeres to questions of this nature contain extremely valuable information about the field.

For Einstein's field equations it is very hard to give a precise meaning to such questions, and even more to find an answer. The equations are nonlinear, but in contrast to nonlinear field theories on Minkowski-space, the field itself determines the spacetime geometry! Even to find "where infinity is" is a nontrivial matter!

The decay behaviour of linear equations is much simpler. Therefore it is natural, in the case of nonlinear field theories on Minkowski space, to ask the question whether solutions exist for which the field becomes small for $t \rightarrow \infty$ and tends to a solution of the linearised equations, the nonlinear effects becoming irrelevant. This leads to a scattering theory in the sense of Lax and Phillips [1].

To follow a similar procedure for Einstein's equations, one has first to formulate in which sense a spacetime becomes asymptotically Minkowskian, and then to establish that there exist solutions having this property. The first problem was posed by Bondi et al. [2] and Penrose [3], leading to the concept of spacetimes admitting null infinity $\mathscr{I}$. A spacetime with this property becomes Minkowskian along null geodesics, and the curvature tensor approaches a solution of the linear spin-two field equations.

The compatibility of these conditions with the field equations remained however rather unclear.

In this paper I examine the asymptotic behaviour of a well-known class of local solutions of Einstein's vacuum field equations, the Einstein-Rosen waves [4, 5]. It 
turns out, that many such solutions exist, which behave asymptotically as the interior of a null cone in Minkowski-space. Technically this means that their future infinity consists of a "piece of $\mathscr{I}^{+}$", which is topologically $S^{2} \times \mathbb{R}$ with future complete generators, which have a regular endpoint $I^{+}$.

Einstein-Rosen waves are solutions of the vacuum field equations admitting two spacelike, hypersurface orthogonal Killing vectors. The usual interpretation considers those solutions as cylindrical gravitational waves [5]. Thus one cannot expect the field to become asymptotically Minkowskian. There is, however, another possibility: Consider the subgroup of the Lorentz group, consisting of boosts in the $t-z$ plane and of rotations in the $x-y$ plane of Minkowski space. In the interior of the null cone of the origin this group defines two spacelike, hypersurface orthogonal killing fields. Clearly, the global action is rather different from cylindrical symmetry.

Einstein-Rosen waves with this asymptotic behaviour of the Killing fields admit a $\mathscr{I}^{+}$and regular $I^{+}$, as described above.

Particular solutions of this type were found and used about 15 years ago to describe "exact solutions for uniformly accelerated particles" by Bonnor and Swaminarayan [6, 7]. In 1963, a paper by Bicák [8] "analizes the radiative properties of Bonnor's and Swaminarayan's solutions by Bondi's method", concluding that it satisfies Bondi's conditions or - in present notation - admits a $\mathscr{I}^{+}$with topology $S^{2} \times \mathbb{R}$. Hence the issue of the existence of radiative solutions admitting such a $\mathscr{I}^{+}$was already solved in 1967, but unfortunately seems to have been overlooked by the workers in the field.

Einstein-Rosen waves in a neighbourhood of $\mathrm{I}^{+}$are, in complete analogy to sourcefree Maxwell fields, determined by Cauchy data on a mass-shell. The regularity of $I^{+}$means that the gravitational field decays completely through $\mathscr{I}^{+}$ and that the limit of the Bondi-mass $(u \rightarrow+\infty)$ vanishes. Most likely, the solutions can also be characterised by an asymptotic, characteristic initial value problem, demanding regular $I^{+}$and prescribing the radiation field with certain symmetries on $\mathscr{I}^{+}$.

The paper is organised as follows. In Sect. 2 conditions on the metric coefficients of a general Einstein-Rosen wave are derived, which imply the existence of a regular $I^{+}$, hence $\mathscr{I}^{+}$. In Sect. 3 it is shown that solutions of the field equations exist, which satisfy these properties.

\section{The Geometry}

Locally Einstein-Rosen waves are described by the metric [5]

$$
e^{2 \gamma-2 \psi}\left(d r^{2}-d t^{2}\right)+r^{2} e^{-2 \psi} d \phi^{2}+e^{2 \psi} d z^{2}
$$

with $\gamma(r, t), \psi(r, t)$. The metric (2.1) is invariantly characterised by the existence of two spacelike hypersurface orthogonal Killing fields $\left(\frac{\partial}{\partial \phi}, \frac{\partial}{\partial z}\right)$ and a condition on the Ricci tensor $\left(R_{\phi}^{\phi}+R_{t}^{t}=0\right)$. If we put $\gamma=\psi=0,(2.1)$ describes Minkowski space in coordinates adapted to a rotational and translational Killing field. 
Motivated by the considerations of Sect. 1, the action of a boost and rotational Killing field on Minkowski space we consider the following metric

$$
g=e^{\lambda}\left(d a^{2}-d b^{2}\right)+a^{2} e^{-\mu} d \phi^{2}+b^{2} e^{\mu} d \chi^{2}
$$

with $\lambda(a, b), \mu(a, b)$. If $\lambda=\mu=0,(2.2)$ describes a part of Minkowski space in coordinates adapted to a rotation and a boost. The metrics (2.1), (2.2) are locally isometric in the sense, that the coordinate transformation

$$
(r+t)=\frac{1}{2}(a+b)^{2}, \quad(r-t)=-\frac{1}{2}(a-b)^{2}
$$

transform (2.1) into the form (2.2).

In the case of Minkowski space we know, "how to extend (2.2) through $a=b=0$ ". The following Lemma shows under which conditions an analogous extension can be made in the general case.

\section{Lemma 1.}

Assumption. 1. $\lambda(a, b), \mu(a, b)$ are realvalued, $C^{\infty}$ functions defined for

$$
0 \leqq a<a_{0}, \quad a \leqq b<b_{0} .
$$

2. $\lambda(0,0)=\mu(0,0)=0, \lambda(0, b)=-\mu(0, b)$.

3. The functions $\hat{\lambda}, \hat{\mu}$ defined by $\hat{\lambda}\left(a^{2}, b^{2}\right)=\lambda(a, b), \hat{\mu}\left(a^{2}, b^{2}\right)=\mu(a, b)$ are $C^{\infty}$ at $(0,0)$, and $\left(0, b^{2}\right)$.

Assertion. The metric (2.2) defined by $\lambda, \mu$ on the manifold

$$
M=\left\{(a, b, \phi, \chi) ; 0<a<a_{0}, a<b<b_{0}, \phi \bmod 2 \pi, \chi \in \mathbb{R}\right\}
$$

has a $C^{\infty}$-extension, such that $M$ is bounded by a null cone $N$ with vertex $p$, and a two dimensional timelike, 2-surface $F$, the "axis" of the $\partial / \partial \phi$-isometry.

Proof. Define coordinates $x, y, z, t$ by

$$
\begin{array}{ll}
x=a \cos \phi & t=b \cosh \chi \\
y=a \sin \phi & z=b \sinh \chi .
\end{array}
$$

Substituting in (2.2), where defined, one gets:

$$
\left(a^{2}=x^{2}+y^{2}, b^{2}=t^{2}-z^{2}>0 \Leftrightarrow t^{2}>z^{2}\right) .
$$

$$
\begin{aligned}
g= & \frac{1}{a^{2}} e^{\lambda}(x d x+y d y)^{2}-\frac{1}{b^{2}} e^{\lambda}\left(z d z-t d t^{2}\right) \\
& +e^{-\mu} \frac{1}{a^{2}}(x d y-y d x)^{2}+\frac{1}{b^{2}} e^{\mu}(z d t-t d z)^{2} .
\end{aligned}
$$

The coefficient of $d x^{2}$ is $\left(x^{2}+y^{2}\right)^{-1}\left\{e^{\lambda} x^{2}+e^{-\mu} y^{2}\right\}$, the one of $d x d y$ is $\left(x^{2}+y^{2}\right)^{-1}\left\{e^{\lambda} 2 x y-e^{-\mu} 2 x y\right\}$.

Because of the assumptions 2), 3), these functions have a $C^{\infty}$ extension into a neighbourhood of $x=y=z=t=0$. The same is true for all other metric coefficients in (2.5). Hence by comparing the range of the $x, y, z, t$ coordinates defined by $M$ one gets the assertion. 
Remark. Obviously one can in the same way give $C^{k}$-versions of this lemma.

Points with $(a, b) \rightarrow(0,0)$ tend to the vertex of the null cone of the extension. The null cone $N$ is approached by points $(a, b) \rightarrow(a, a)$, the axis of the rotation by points $(a, b) \rightarrow(0, b)$.

Note that to get the null cone as a boundary one needs $\phi \bmod 2 \pi$ and $\chi \in \mathbb{R}$ !

From general arguments (regular $b$-boundary) one knows that the axis and the null cone are determined uniquely by the original spacetime.

We turn to the behaviour of (2.2) for large $a, b$. Suppose $\lambda, \mu$ are defined for $0<a,<b, b_{0}<b<\infty$. Guided by the inversion in Minkowski space we transform coordinates:

$$
\tilde{a}=\frac{-a}{a^{2}-b^{2}}, \quad \tilde{b}=\frac{-b}{a^{2}-b^{2}} .
$$

Using $\tilde{a}+\tilde{b}=-(a-b)^{-1}, \tilde{a}-\tilde{b}=-(a+b)^{-1}$, one gets immediately

$$
\begin{gathered}
g=\left(\tilde{a}^{2}-\tilde{b}^{2}\right)^{-2}\left[e^{\tilde{\lambda}}\left(d \tilde{a}^{2}-d \tilde{b}^{2}\right)+\tilde{a}^{2} e^{-\tilde{\mu}} d \phi^{2}+\tilde{b}^{2} e^{\tilde{\mu}} d \chi^{2}\right], \\
\tilde{\lambda}(\tilde{a}, \tilde{b})=\lambda\left(-\frac{\tilde{a}}{\tilde{a}^{2}-\tilde{b}^{2}},-\frac{\tilde{b}}{\tilde{a}^{2}-\tilde{b}^{2}}\right),
\end{gathered}
$$

and $\tilde{\mu}$ correspondingly. Hence the rescaled metric $\tilde{g}=\left(\tilde{a}^{2}-\tilde{b}^{2}\right)^{2} g$ is again of the Einstein-Rosen form (2.2)!

Now we can establish the following:

\section{Lemma 2.}

Assumption. $\mu(a, b), \lambda(a, b)$ are defined for $0<a<b, b_{0}<b<\infty$ and $\tilde{\mu}, \tilde{\lambda}$ by (2.8). Suppose $\tilde{\mu}, \tilde{\lambda}$ satisfy the assumption of Lemma 1.

Assertion. The spacetime (2.2) defined by $\lambda, \mu$ admits $\mathscr{I}^{+}$according to Penrose and $\mathscr{I}^{+}$and $I^{+}$are $C^{\infty}$.

Proof. The rescaled spacetime $\tilde{g}=\Omega^{2} g$ with $\Omega=\left(\tilde{a}^{2}-\tilde{b}^{2}\right)$ admits an extension through a null cone with vertex because $\tilde{\mu}, \tilde{\lambda}$ satisfy the assumption of Lemma 1 . $d \Omega=2 \tilde{a} d \tilde{a}-2 \tilde{b} d \tilde{b} \neq 0$ for $a=b>0$, hence $\tilde{N}$ satisfies the defining conditions of $\mathscr{I}^{+}$. Furthermore the generators have also a regular endpoint $I^{+}$.

Remark. We have chosen a conformal factor such that the rescaled metric $\tilde{g}$ still has $\frac{\partial}{\partial \phi}, \frac{\partial}{\partial \chi}$ as Killing fields. This is the reason, why it is so simple to do the extension.

\section{The Field Equations}

The structure of the vacuum field equations for Einstein-Rosen waves is very simple. Because of the symmetry one has only two independent variables. Furthermore - as in the case of Weyl solutions - one of the metric coefficients is determined by a linear equation, the other by integration. 
For the purpose of the following analysis null coordinates are introduced for the metric (2.2)

$$
u=b-a, \quad v=b+a .
$$

The metric is then

$$
g=-e^{\lambda} d u d v+\frac{1}{4}(v-u)^{2} e^{-\mu} d \phi^{2}+\frac{1}{4}(v+u)^{2} e^{\mu} d \chi^{2} .
$$

The vacuum field equations are satisfied provided it holds:

$$
\begin{gathered}
\left\{\partial_{u} \partial_{v}+\frac{1}{v^{2}-u^{2}}\left(v \partial_{u}-u \partial_{v}\right)\right\} \mu=0, \\
\partial_{v} \lambda=\frac{1}{4 v}\left(v^{2}-u^{2}\right)\left(\partial_{v} \mu\right)^{2}-\frac{u}{v} \partial_{v} \mu, \\
\partial_{u} \lambda=-\frac{1}{4 u}\left(v^{2}-u^{2}\right)\left(\partial_{u} \mu\right)^{2}-\frac{v}{u} \partial_{u} \mu .
\end{gathered}
$$

The linear equation (3.3) for $\mu$ is the wave equation for the metric (3.2) with $\lambda=\mu$ $=0$. Hence any solution of $\square \mu=0$ on Minkowski space which depends only on $(a, b)$ solves (3.3). If $\mu$ is given, $\lambda$ can be determined from (3.4) or (3.5) by integration. (3.3) is the integrability condition for (3.4). (3.5).

Consider an example: $\mu=(u v)^{-1}$ solves (3.3). For $\lambda$ one gets the equation

$$
\begin{gathered}
\partial_{v} \lambda=+\frac{1}{4 v}\left(v^{2}-u^{2}\right)\left(\frac{1}{u v^{2}}\right)-\frac{u}{v}\left(\frac{1}{u v^{2}}\right) \\
\lambda=-\frac{1}{8 u^{2} v^{2}}+\frac{1}{16 v^{4}}-\frac{1}{2 v^{2}}+f(u) .
\end{gathered}
$$

To satisfy the regularity condition at the axis $(\lambda(v, v)=-\mu(v, v))$ one chooses $f(u)=$ $+\frac{1}{16 u^{4}}-\frac{1}{2 u^{2}}$, hence

$$
\lambda=-\frac{1}{8 u^{2} v^{2}}+\frac{1}{16 v^{4}}-\frac{1}{2 v^{2}}+\frac{1}{16 u^{4}}-\frac{1}{2 u^{2}} .
$$

We can now show:

Theorem. There exist $C^{\infty}$ Einstein-Rosen waves admitting $\mathscr{I}^{+}$, which is topologically $S^{2} \times \mathbb{R}$ with future complete generators and a $C^{\infty}-I^{+}$.

Proof. Suppose $\tilde{f}(\tilde{x}, \tilde{y}, \tilde{z}, \tilde{t})$ is a $C^{\infty}$ solution of the wave equation

$$
+\partial_{\tilde{t}}^{2} \tilde{f}+\partial_{\tilde{x}}^{2} \tilde{f}+\partial_{\tilde{y}}^{2} \tilde{f}+\partial_{\tilde{z}}^{2} \tilde{f}=0
$$

which is invariant under rotations in the $\tilde{x}, \tilde{y}$-plane and boosts in the $\tilde{z}-\tilde{t}$ plane. Such solutions can be determined by a characteristic initial value problem [9] with data on the null cone of $(\tilde{x}, \tilde{y}, \tilde{z}, \tilde{t})=0$. Passing to $(\tilde{a}, \tilde{b})$ we have a function $f(\tilde{a}, \tilde{b})$. The function

$$
\mu(u, v)=(u v)^{-1} \tilde{f}(\tilde{a}(u, v), \tilde{b}(u, v))
$$


satisfies Eq. (3.3) due to the behaviour of the wave equation under conformal rescaling. Put

$$
\tilde{u}=\tilde{b}-\tilde{a}, \quad \tilde{v}=\tilde{b}+\tilde{a} \Leftrightarrow \tilde{u}=\frac{1}{v}, \quad \tilde{v}=\frac{1}{v} .
$$

Rewriting (3.4), (3.5) with the variables $\tilde{u}, \tilde{v}$ using

$$
\mu(u, v)=(u v)^{-1} \cdot \tilde{f}(\tilde{u}(v), \tilde{v}(u))
$$

one gets

$$
\begin{aligned}
-\partial_{\tilde{u}} \tilde{\lambda} & =\frac{1}{4}\left(\tilde{v}^{2}-\tilde{u}^{2}\right) \tilde{u}\left[\tilde{f}+\tilde{u} \partial_{\tilde{u}} \tilde{f}\right]^{2}+\tilde{u}\left[\tilde{f}+\tilde{u} \partial_{\tilde{u}} \tilde{f}\right], \\
\partial_{\tilde{v}} \tilde{\lambda} & =\frac{1}{4}\left(\tilde{v}-\tilde{u}^{2}\right) \tilde{v}\left[\tilde{f}+\tilde{v} \partial_{\tilde{v}} \tilde{f}\right]^{2}-\tilde{v}\left[\tilde{f}+\tilde{v} \partial_{\tilde{v}} \tilde{f}\right] .
\end{aligned}
$$

Note that the right-hand side of (3.10), (3.11) is $C^{\infty}$ in $\tilde{u}, \tilde{v}$ at $\tilde{u}=\tilde{v}=0$. Using (3.10) we define $\tilde{\lambda}$ by

$$
\tilde{\lambda}(\tilde{u}, \tilde{v})=\int_{v}^{u} \partial_{\tilde{u}} \tilde{\lambda}(\tilde{u}, \tilde{v}) d \tilde{u}-\tilde{\mu}(\tilde{v}, \tilde{v})
$$

This implies that $\tilde{\lambda}(\tilde{u}, \tilde{v})$ is $C^{\infty}$ at $\tilde{u}=\tilde{v}=0$ and at $\tilde{u}=0$. To apply Lemma 2 it remains to show that $\tilde{\lambda}(\tilde{a}, \tilde{b}), \tilde{\mu}(\tilde{a}, \tilde{b})$ are $C^{\infty}$ functions of $\tilde{a}^{2}, \tilde{b}^{2}$ at the axis of the rotation. For $\tilde{\mu}$ this is true because $\tilde{\mu}$ is $\left(\tilde{a}^{2}-\tilde{b}^{2}\right) \cdot \tilde{f}$, where $\tilde{f}$ is a $C^{\infty}$ solution of the wave equation (3.6). For $\tilde{\lambda}$, this can be shown as follows: (3.4), (3.5) imply:

$$
\begin{aligned}
\left(b^{2}-a^{2}\right) \partial_{a} \lambda= & \frac{1}{2} a b^{2}\left[\left(\partial_{a} \mu\right)^{2}+\left(\partial_{b} \mu\right)^{2}\right]-b \partial_{a} \mu+a b \partial_{b} \mu \\
& -\frac{1}{2} a^{2} b \partial_{a} \mu \partial_{b} \mu-a^{2} \partial_{a} \mu+a b \partial_{b} \mu .
\end{aligned}
$$

Suppose $\tilde{\mu}(\tilde{a}, \tilde{b})$ is analytic at $\tilde{a}=\tilde{b}=0$, then we have an expansion

$$
\tilde{\mu}=\sum_{n, m=0}^{\infty} \alpha_{m m} \tilde{a}^{2 n} \tilde{b}^{2 m} \text {. }
$$

Hence

$$
\begin{aligned}
\mu(a, b) & =\sum \alpha_{m n}\left(\frac{a}{b^{2}-a^{2}}\right)^{2 m}\left(\frac{b}{b^{2}-a^{2}}\right)^{2 n} \\
& =\sum \alpha_{n n}\left[\frac{a}{b^{2}\left(1-\frac{a^{2}}{b^{2}}\right)}\right]^{2 m}\left[\frac{b}{b^{2}\left(1-\frac{a^{2}}{b^{2}}\right)}\right]^{2 n} .
\end{aligned}
$$

This implies that the right-hand side of (3.13) is an odd function of $a$ and an even function of $b$. Hence $\lambda$ is an even function of $a$ and $b$. Going back to $\tilde{a}, \tilde{b}$, this gives that $\tilde{\lambda}(\tilde{a}, \tilde{b})$ has an analytic expansion in $\tilde{a}^{2}, \tilde{b}^{2}$. Hence $\tilde{\mu}, \tilde{\lambda}$ satisfy the assumption of Lemma 2, which completes the proof in the analytic case. If $\mu$ is only $C^{\infty}$, we argue as follows. The condition " $\tilde{\lambda}$ is smooth as a function of $\tilde{a}^{2}, \tilde{b}^{2}$ " means that certain derivatives of $\tilde{\lambda}$ at $(\tilde{a}, \tilde{b})=0$ vanish. The relations between the partial derivatives of $\lambda, \mu$ given by (3.13) are the same as in the analytic case, hence the critical derivatives vanish. 


\section{Conclusions}

The last two sections showed, how one can construct Einstein-Rosen waves with a regular $I^{+}$and $\mathscr{I}^{+}$. Choosing simple functions $\mu(a, b)$ one can even give the metric explicitely. To use these spacetimes as nontrivial examples of Bondi et al. and Penrose's radiation theory one has to show that these solutions are radiating. A systematic investigation of the radiation properties will be done in a separate paper. In the following, I give just some plausibility considerations, using standard radiation descriptions in the Penrose notation.

$\mathscr{I}^{+}$is radiative, if the news function $\sigma^{0}$ - Penrose notation - does not vanish. Because the news is rather complicated to calculate, one can proceed as follows. The $C^{\infty}$ regularity of $I^{+}$implies that $\psi_{0}^{0}, \psi_{1}^{0}, \psi_{2}^{0}, \psi_{3}^{0}, \psi_{4}^{0}$, vanish at $I^{+}$. The propagation equation for those quantities $[10,(4.4)-(4.10)]$ show that if any of those quantities is not zero at a point $p$ near $I^{+}$, the $\dot{\sigma}^{0}$ cannot be identically zero on the generator through $p$ between $p$ and $I^{+}$. Hence to conclude that a solution is radiative, one has just to establish $\partial_{e} C^{a}{ }_{b c d} \neq 0$ on $\mathscr{I}^{+}$. This can be done in particular examples. The vanishing of the $\psi_{i}^{0}$ at $I^{+}$implies further that the limit of the Bondi-mass vanishes if one approaches $I^{+}$.

There is strong evidence that the only Einstein-Rosen wave with a regular $I^{+}$ and no radiation near $I^{+}$is Minkowski space! To prove a uniqueness theorem of this nature one would have to extend Friedrich's [11] treatment of the asymptotic, characteristic initial value problem, to include the case of giving data at $I^{+}$and on $\mathscr{I}^{+}$near $I^{+}$. More directly, this can probably be derived using the Eqs. (3.10) and (3.11).

So far the solutions were only considered near $I^{+}$. Choosing a solution of $\square \mu=0$ invariant under a boost and a rotation one can try to solve for $\lambda$ as long as $\mu$ is defined. This way one will get a more systematic description and more general solutions of the Bonnor-Swaminarayan type. In particular, the $C$-metric, for which Ashtekar and Dray showed recently the existence of $\mathscr{I}[12]$ should also be in this class.

Finally a remark about the regularity of $I^{+}$. Asymptotics was invented for isolated systems which have no regular $I^{+}$, if material sources are present. There should, however, also exist asymptotically flat, source-free spacetimes, i.e. gravitational waves coming in at $\mathscr{I}^{-}$and going out at $\mathscr{I}^{+}$. In such a situation one expects $I^{-}, I^{+}$to be regular, because the whole field is decaying through $\mathscr{I}^{+}$. The solutions described, have the essential properties of such a spacetime near $I^{+}$.

There are, however, no Einstein-Rosen waves with regular $\mathscr{I}^{+}, \mathscr{I}^{-}$because any non-constant solution of $\square \mu=0$ with the boost-rotation symmetry is singular somewhere.

Acknowledgements. I would like to thank H. Friedrich, H.-J. Seifert, M. Streubel, and the Munich Relativity group for hepful discussions.

\section{References}

1. Lax, P.P., Phillips, R.S.; Scattering theory. New York: Academic Press 1967

2. Bondi, H., Burg, M.G.J., van der, Metzner, A.W.K. : Proc. R. Soc. (London) A 269, 21 (1962)

3. Penrose, R. : Phys. Rev. Lett. 10, 2 (1963)

4. Einstein, A., Rosen, N.: J. Franklin Inst. 223, 43 (1937) 
5. Synge, J.W.: Relativity. The general theory. Amsterdam: North-Holland Publishing Company 1971

6. Bonner, W.B., Swaminarayan, N.S.: Z. Physik 177, 240 (1964)

7. Swaminarayan, N.S.: Commun. Math. Phys. 2, 59 (1960)

8. Bicák, J.: Proc. R. Soc. A 302, (1968)

9. Friedlander, G.: The wave equation in curved spacetime. Cambridge: Cambridge University Press 1975

10. Newman, E.T., Penrose, R.: Proc. Soc. (London) A305, 175 (1968)

11. Friedrich, H.: Preprint, Hamburg

12. Ashtekar, A., Dray, T.: Preprint, Clermont-Ferrant

Communicated by R. Geroch

Received July 18, 1980 